\title{
Beyond gastritis and before cancer: the strange case of Ménétrier's disease
}

\author{
Giuseppe Famularo • Maria Rosaria Sajeva • \\ Laura Gasbarrone
}

Received: 18 August 2010/ Accepted: 23 September 2010/Published online: 14 October 2010

(C) SIMI 2010

A 73-year-old man complained of epigastric discomfort, dysphagia, and weight loss of $8 \mathrm{~kg}$ over the prior 2 months; he denied hematemesis, hematochezia, melena, diarrhea, fever, or any other symptoms. The patient had severe leg weakness with intermittent bladder and bowel incontinence caused by a spinal cord compressing D6 fracture, hypertension, a repaired abdominal aortic aneurysm, and recurrent venous thromboembolism, for which a filter was inserted in the inferior cava vein. There was neither personal and family history of allergy, nor any gastrointestinal disorders, including cancer and peptic ulcer disease.

The physical examination was unrevealing: the vital signs were normal and stable, and laboratory studies were normal except for a hemoglobin $9.8 \mathrm{~g} / \mathrm{dl}$, hematocrit $38.8 \%$, total protein $6.9 \mathrm{~g} / \mathrm{dl}$, and albumin $3.0 \mathrm{~g} / \mathrm{dl}$. A computed tomography of the abdomen showed thickened gastric walls, an air fluid level in the lower esophagus, and abundant intraperitoneal fluid around the liver and the spleen, and in the pelvis. Endoscopy of the upper gastrointestinal tract revealed an almost complete obstruction of the gastric lumen by giant folds of the mucosa with rugae closely resembling cerebral convolutions with a finely nodular (cobblestone) appearance (Fig. 1). There was abundant and thick mucus, and an ulcerated mass was partially obstructing the pyloric outlet (not shown).

Microscopy of multiple biopsies of gastric giant folds showed mucosal and foveolar hypertrophy, elongated crypts, dilated gastric glands, some of which were lined by a large number of mucous cells, and glandular cysts extending into the submucosa. There was no evidence of

G. Famularo $(\varangle) \cdot$ M. R. Sajeva · L. Gasbarrone

Department of Internal Medicine, San Camillo Hospital,

Circonvallazione Gianicolense, 00152 Rome, Italy

e-mail: gfamularo@scamilloforlanini.rm.it mucosal or submucosal inflammation, ulcers, or granulomata and no eosinophilic infiltration throughout the entire thickness of specimens. The stains for mycobacteria and fungi were negative, and cultures grew no organisms. We considered all these histological findings diagnostic of Ménétrier's disease. Microscopy of biopsies of the pyloric mass showed a poorly differentiated carcinoma.

Proton pump inhibitors, corticosteroids, and parenteral nutrition were started; the patient refused surgery and was discharged to a hospice.

Hypertrophic gastropathy can be caused by a wide spectrum of disorders ranging from Zollinger-Ellison syndrome to sarcoidosis, syphilis, and eosinophilic gastroenteritis; however, giant gastric folds associated with foveolar hyperplasia are more likely due to Ménétrier's disease than other causes of hypertrophic gastropathy [1].

Ménétrier's disease is a rare disease, characterized by enlargement of the gastric mucosal folds that primarily affect the body of the stomach, whereas the gastric antrum is usually saved [1]. Microscopy shows proliferation of the gastric glands with preservation of nuclear polarity and cystic dilatation of their basilar portion. In extensive cases, the mucous-secreting cells are present in the glands deep in the mucosa and replace the normal chief and parietal cells. Inflammation in the lamina propria is modest although the enlarged folds are subject to erosion and can show superficial ulcers with granulation tissue and an influx of neutrophils into the lamina propria. A patchy accumulation of eosinophils in the lamina propria is an under appreciated feature of Ménétrier's disease even though eosinophils are sometimes the predominant inflammatory cells; lamina propria lymphocytes may also be mildly increased. The muscularis mucosa is thickened and disorganized, with strands of smooth muscle extending into the lamina propria, as well as prolapse of the enlarged folds. 




Fig. 1 The typical endoscopic appearance of gastric mucosal hypertrophy in Ménétrier's disease is seen. The stomach lumen is almost completely obstructed by giant gastric folds with rugae closely resembling cerebral convolutions, yielding a finely nodular (cobblestone) appearance; mucus is abundant and thick. Expansion of mucosal enlarged folds is most marked in the body and fundus with the antrum usually spared

The etiology of Ménétrier's disease remains unknown, and no definite diagnostic criteria have been established, but a close clinical and morphological correlation with the endoscopic findings is required [1]. It is noteworthy that routine endoscopic superficial mucosal biopsies may not be informative, and deeper or full-thickness biopsies of the gastric mucosa are needed in some cases.

Many patients have subtle clinical features and can present across a broad spectrum of phenotypes; however, most of them have a unique constellation of signs and symptoms that include abdominal pain, nausea and vomiting, anemia (due to gastric blood loss), hypochlorhydria (due to markedly reduced, or absence of parietal cells), and edema of peripheral tissues (due to leakage of protein across the gastric mucosa [1]). The resulting decreased intravascular volume is thought to favor the occurrence of venous thromboembolism [1]. The average age at diagnosis is 55 years, and the disease seems to be more common in men than in women [1]. Ménétrier's disease is a progressive disorder in adults; however, a cytomegalovirus-related variant that resolves spontaneously has been described in childhood [2]. Familial clusters of Ménétrier's disease have been reported [3].

One important point is that Pierre Ménétrier [4] did not formulate a syndrome, but rather reported the post-mortem description of two patients showing extraordinary gastric abnormalities that were associated with the development of gastric carcinoma. He also noted the similarity of the large gastric folds to those of the third stomach of ruminants, i.e. the omasum.

There is general agreement about the potential to evolve into a malignancy since the first report in 1888; however, the association with cancer has so far been described only in case reports and small series of patients. Despite this limitation, Ménétrier's disease should be viewed as a precancerous condition, and patients should be viewed at risk to develop gastric cancer even though the magnitude of this risk is not definite.

The mechanisms of carcinogenesis in Ménétrier's disease are unknown. Formerly, a disorder with an uncertain etiology, Ménétrier's disease is now believed to result from enhanced epithelial growth factor receptor (EGFR) signaling in the gastric mucosa, which is linked to local overproduction of transforming growth factor (TGF)- $\alpha$ [5]. Both TGF- $\alpha$ and EGFR are expressed in the normal gastric mucosa; however, several experimental and clinical observations suggest that overproduction of TGF- $\alpha$ in the stomach might contribute to the pathogenesis of Ménétrier's disease. For example, patients with Ménétrier's disease have increased TGF- $\alpha$ expression in the mucous cell compartment of the stomach [5]; transgenic mice that overexpress rat TGF- $\alpha$ in the stomach exhibit virtually all the features of Ménétrier's disease [5], and the Ménétrier's phenotype has been observed in transgenic mice expressing human TGF- $\alpha$ in the stomach [5]. Increased TGF- $\alpha$ expression has also been found in the involved gastric mucosa in a childhood case of cytomegalovirus-associated Ménétrier's disease [5]. Increased oxidative DNA damage and increased mutagenicity of gastric juice have been demonstrated in the subset of patients with Ménétrier's disease who are also colonized by Helicobacter pylori [5].

There is no gold-standard treatment strategy for Ménétrier's disease, and no evidence-based guideline is currently available. Partial or total gastrectomy is recommended for cases in which there is concern over the development of cancer or for those with debilitating disease [1]. Empiric treatment with anticholinergic therapy, octreotide, acid suppression, and prednisone, as well as eradication of Helicobacter pylori, has provided no consistent benefit [1].

\section{Conflict of interest None.}

\section{References}

1. Wolfsen HC, Carpenter HA, Talley NJ (1993) Ménétrier's disease: a form of hypertrophic gastropathy or gastritis? Gastroenterology 104:1310-1319

2. Occena RO, Taylor SF, Robinson CC, Sokol RJ (1993) Association of cytomegalovirus with Ménétrier's disease in childhood: report of two new cases with a review of literature. J Pediatr Gastroenterol Nutr 17:217-224 
3. Larsen B, Tarp U, Kristensen E (1987) Familial giant hypertrophic gastritis (Ménétrier's disease). Gut 28:1517-1521

4. Ménétrier P (1888) Des polyadenomesgastriques et de leurs rapports avec le cancer de l'estomac. Arch Physiol Norm Pathol $1(32-55): 236-262$
5. Coffey RJ, Washington MK, Corless CL, Heinrich MC (2007) Ménétrier disease and gastrointestinal stromal tumors: hyperproliferative disorders of the stomach. J Clin Invest 117:70-80 